\title{
Characterization of crack propagation during sonic IR inspection
}

\author{
Jacob Kephart, John Chen, Hong Zhang \\ Rowan University, Mech Eng, 201 Mullica Hill Rd, Glassboro, NJ USA 08028
}

\begin{abstract}
Sonic IR is an emerging, thermal-based, nondestructive evaluation (NDE) technique. Typically a short burst of high power acoustical energy is injected into an object being studied and certain types of defects heat up and is detected using a thermal imaging camera. This inspection technique is very fast, lasting only a few seconds for total inspection time. However due to many uncertainties in the inspection process it has yet to be adopted widely by industry. There are many unknown parameters governing sonic IR, which need to be understood before it becomes a widely used NDE technique. This paper shows that under certain conditions cracks can grow when subjected to the sonic IR technique. We also examine the effects of various experimental parameters on the technique.
\end{abstract}

Keywords: sonic IR, NDE, thermal, inspection, thermosonics, crack growth

\section{INTRODUCTION}

Sonic IR currently as implemented is a thermal-based NDE technique first described by Thomas and colleagues in 2000 [1,2]. The method utilizes an ultrasonic transducer, which is capable of high power outputs, typically up to $1 \mathrm{kw}$, and vibrates at low ultrasonic frequencies of $20 \mathrm{kHz}$ or $40 \mathrm{kHz}$. It is thought that the induced vibrations cause frictional heating at crack location sites which can be viewed as regions of increased temperature when viewed through a thermal imaging camera. The typical inspection time is on the order of only a few seconds. This method has been applied to cracks, delaminations, and disbands, and has been successful with a wide range of materials. Furthermore, a similar method, which also utilizes lower frequency ultrasound and a lock-in technique is capable of detecting cracks, defective shrink fits, entrapped air in adhesives, non-cured adhesives, and kissing bonds [3].

While the benefits of this method are easily noticed, such as its speed of detection and its ability to detect defects that are difficult for other methods, there remain many uncertainties with sonic IR. Some of the unknown parameters for successful defect identification are minimum time of energy application, minimum sonic power, required coupling force, effect of backing material, fixturing, and window of inspection.

The goal of this paper is to demonstrate conditions under which a small artificial crack will propagate when subjected to the sonic IR inspection technique. A variety of backing materials and other inspection parameters are also investigated.

\section{METHODOLOGY}

\subsection{Sample Design}

The goal of the experiment is to understand the relationship between sonic energy and the amount of crack growth within a test sample. A standard sample had to be chosen so that sequential tests were on nearly identical specimens. Each sample would be subjected to a certain load history so that all parameters governing the crack growth were consistent. 
The sample design selected was from ASTM standard E647 [4]. This standard utilizes an eccentrically loaded single edge tension specimen (ESET) as seen in Fig. 1.

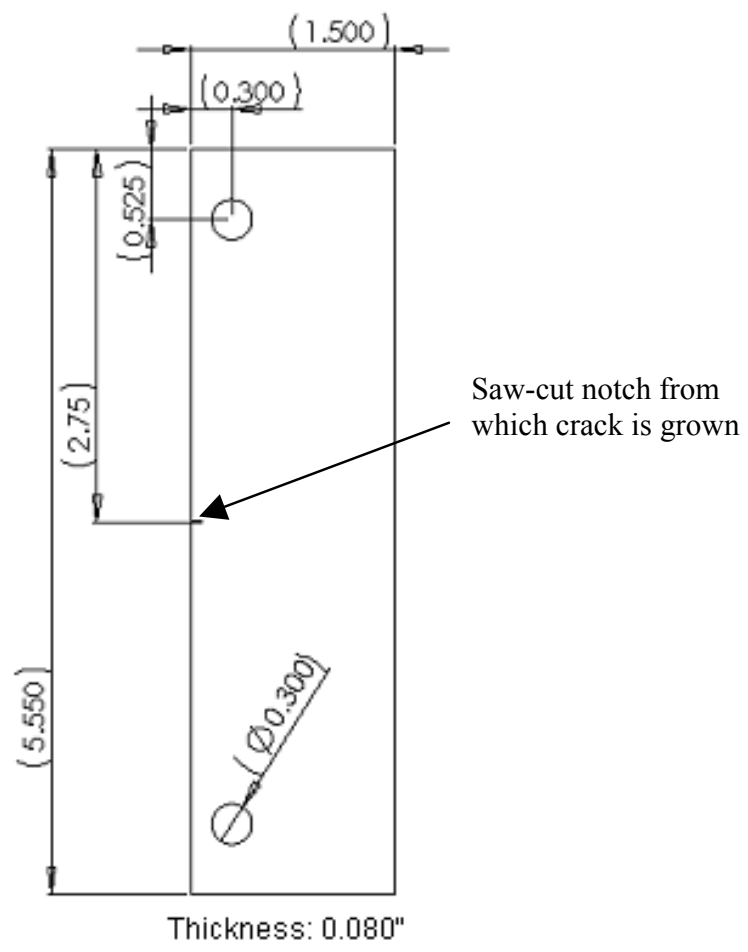

Figure 1. ESET fatigue crack sample dimensions.

This sample shape was chosen over the compact tension (CT) specimen due it is longer profile and larger region for viewing with an optical traveling microscope. This specimen was fabricated from 2024-T3 aluminum chosen for its known fatigue properties. The sample had a jewelers saw notch located at onehalf the length of the sample (see Fig. 1). The cut was through thickness with a width of 0.010 inches, and length of cut was a nominal 0.075 inches. The cut length was chosen so that an initial $\Delta k$ stress intensity factor was around $7 \mathrm{ksi} \cdot \sqrt{i n c h}$ with a terminal $\Delta k$ of $9 \mathrm{ksi} \cdot \sqrt{i n c h}$. These stress intensity factors were achieved with a load ratio of $\mathrm{R}=0.1$.

The stress intensity factor can be calculated with the following formulae for the shape governed by the ESET specimen in ASTM standard E647 [4].

$$
\begin{gathered}
\alpha=\frac{a}{W} \\
G=3.97-10.88 \alpha+26.25 \alpha^{3}-38.9 \alpha^{3}+30.15 \alpha^{4}-9.27 \alpha^{5} \\
F=\alpha^{1 / 2}[1.4+\alpha][1-\alpha]^{-3 / 2} G \\
\Delta K=\left[\frac{\Delta P}{B \sqrt{W}}\right] F
\end{gathered}
$$


Where

$a$ - Crack length plus the notch length in inches

$W$ - Width of the specimen in inches

$\Delta P$ - Max load minus minimum load in kilo-pounds

$B$-Thickness of specimen in inches

The specimen was loaded in a MTS machine with a force set point of $329.4 \mathrm{lbs}$ and amplitude of 269.5lbs and was varied sinusoidally at a frequency of $20 \mathrm{~Hz}$. The crack length was measured and recorded at given intervals using a traveling microscope with a resolution of 0.0001 inches. Cracking was complete when the crack plus notch length was a nominal 0.135 inches. Three samples were created with identical load histories for use in the sonic experiments.

\subsection{Sonic crack growth test setup}

The purpose of this test is to determine if conditions exist which can cause further damage in the form of crack extension. The samples were subjected to sonic IR inspection under various conditions.

The sonic IR inspection setup included a Branson ultrasonic welder as the sonic source. The power supply is a Branson $910 \mathrm{M}$ capable of $1 \mathrm{kw}$ power output and this is coupled to a Branson GK5 handgun converter. The handgun converts the electrical signal to mechanical motion at a frequency of $20 \mathrm{kHz}$. To maintain a constant coupling force, a horizontal rig was fabricated to hold the handgun (Fig. 2.). This rig clamps the gun in place by its external casing, while still allowing its proper operation.

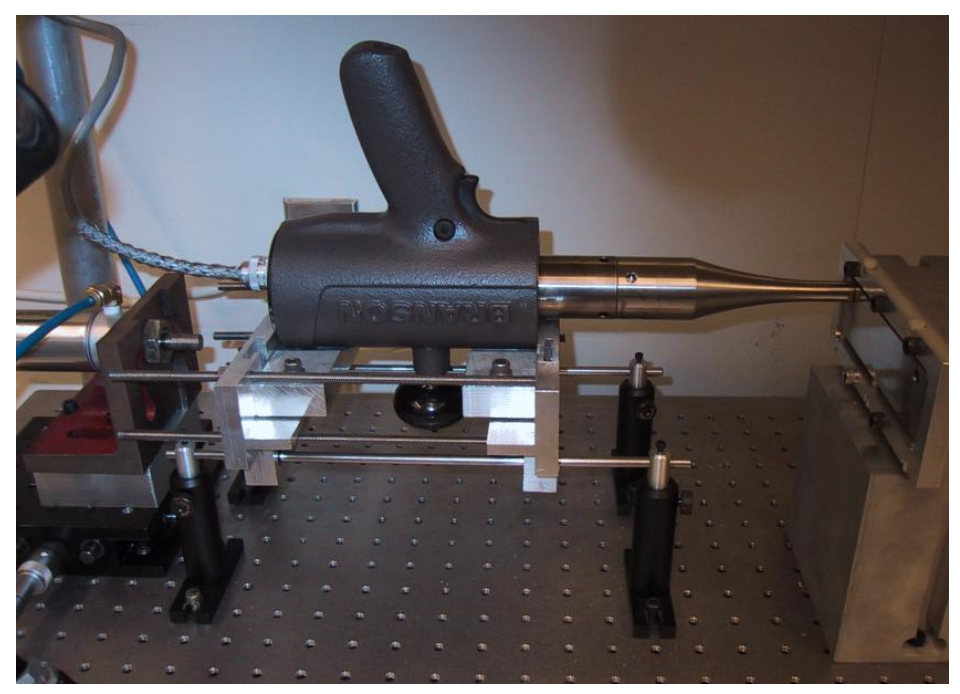

Figure 2. The GK5 handgun secured in rig.

A 1-inch-bore pneumatic piston-cylinder was attached to the rear of the rig so that the amount of force being applied is $\sqrt{1 / 2} \pi *$ pressure (with pressure expressed in psi). Pneumatic pressure is regulated from the source to a pressure less than 30psi. The air then passes through a needle valve into the supply side of a 5 port pneumatic switch as seen in Fig. 3. The exit of the switch supplying pressure to the piston-cylinder has another needle valve through which air passes before reaching the piston-cylinder. The first needle valve is used to regulate the flow of air to the switch. Allowing full flow will result in harsh motion of the handgun and uncontrolled spikes in pressure. The second needle valve's purpose is to isolate the 
pneumatic piston-cylinder from the rest of the air system when the desire pressure has been reached. Without this valve in place, it is possible for pressures within the system to vary and increase with time. We have found that small variations in pressure exerted on the handgun can cause significant variations in the experiment. Isolating the pneumatic piston-cylinder after the desired pressure has been reached minimizes this effect.

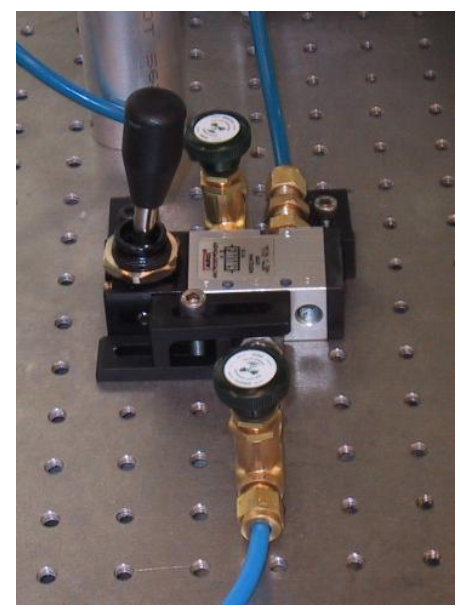

Figure 3. Pneumatic valves and switch to control pressure supplied to pneumatic cylinder.

The horn tip of the handgun has been modified so that it is able to remain securely in place on the sample without slipping or traveling across the sample. The tip has been cut back by 0.050 inches so that a protrusion of 0.300 inches in diameter remains, as seen in Fig. 4. This allows the horn tip to rest inside either of the loading holes of the sample. Since fixturing is typically a problem with sonic IR, using this relieved design limits motion in all direction except the desired longitudinal vibration. This limited motion is at the cost of only having the ability to insonate the test sample at one unique location.

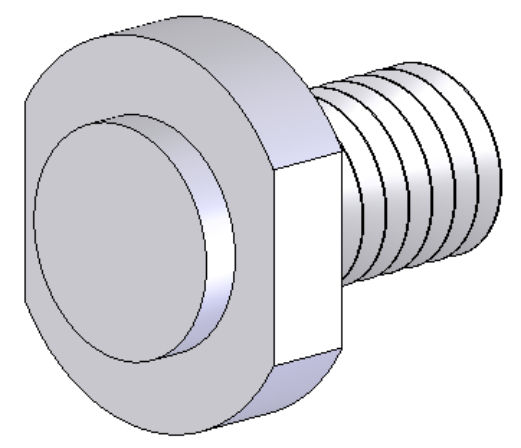

Figure 4. Modified horn tip to disallow slipping across surface.

The sample being tested was secured in a test bed template (Fig. 5) to reduce gross motion, which we have occasionally observed with this technique. The template itself was constructed of aluminum with recesses at one end to allow the sample to be easily removed. Nylon screws were placed on the top and bottom of the sample template to make sure the sample did not come in contact with the edge of the holder during testing. The screws were adjusted to just contact the sample to ensure gross vertical motion was not 
allowed. The back-side of the sample was isolated from contact with the aluminum template through the use of a backing material, which is described in detail below. This sample holder was designed to allow for use with 0.125 -inch-thick backing material and can hold ESET samples of thicknesses 0.080 inches to .250 inches.

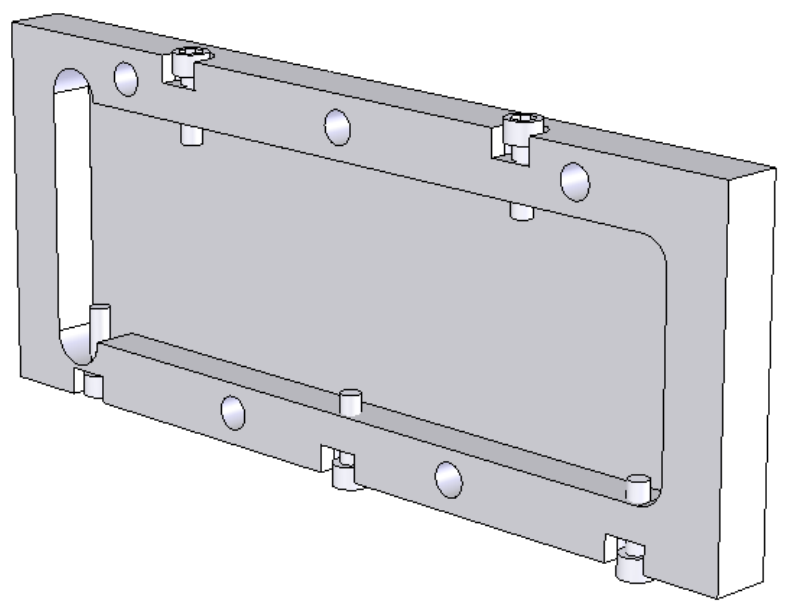

Figure 5. Sample template holder that limits motion of sample during testing.

The sample template (or holder) is securely mounted in the orientation shown in Fig. 5 to a large block of aluminum that serves a base. This base is securely mounted to an optical table along with the handgun rig and pneumatic piston-cylinder.

For the crack growth tests, the sample backing material is limited to a 0.5 -inch wide vertical strip the contact location of the horn and no additional backing material under the rest of the sample. Having a full backing material that spans the entire sample causes a reduced crack detection signal. Fig. 6 exemplifies this with the difference in detection signal for a full backing material of ultra high temperature polyurethane (UHTP). The full backing material shows a peak temperature rise to $30.8 \mathrm{C}$ vs. $34.1 \mathrm{C}$ for the strip of backing material from an ambient temperature of $26.0 \mathrm{C}$. For this particular case the temperature increase was $8.1 \mathrm{C}$ for the strip backing and only $4.8 \mathrm{C}$ for the full backing.
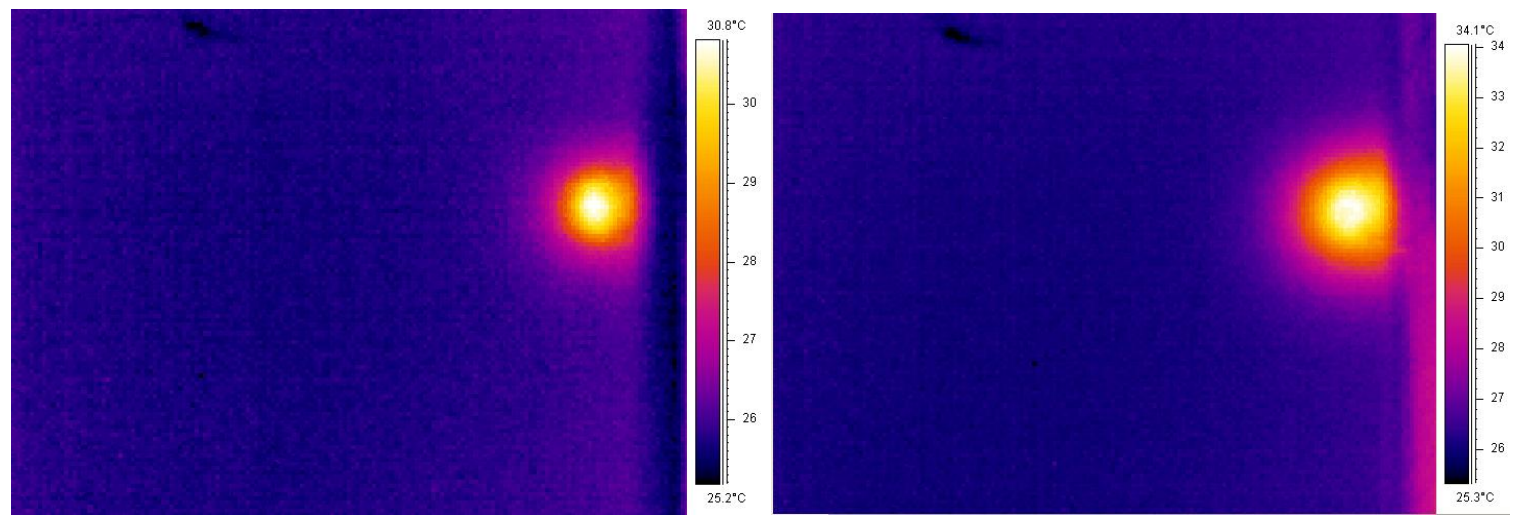

Figure 6. (Left) Sonic IR test using a full piece of backing material. (Right) Sonic IR test using only a strip of backing material. 
The procedure for the sonic IR crack growth test included setting the insonation time interval, setting the pneumatic pressure, insonating the sample, then recording peak power output and total energy output from the power supply, and optically measuring the crack length.

\subsection{Backing material test setup}

The purpose of this test is to compare the effects of various materials for use as backing materials when inspecting a small object using the sonic IR method. This test utilized all the previous equipment however instead of using a cracked sample, testing was conducted on a 0.125-inch-thick, ESET sample without a notch or crack. The measurements made were related to the performance of the ultrasonic power source under various test conditiions. Pressure was controlled in the same manner as previously mentioned. All backing material in this test was rectangular in nature to fit within the sample template holder.

The materials tested included, UHTP, high temperature silicone, cork, chlorinated polyvinyl chloride (CPVC), high-density polyethylene (HDPE), 30-durometer neoprene, and 80-durometer neoprene. Two tests were performed with these materials. The first was to test for consistency of coupling. In these tests the pressure was set, and the test specimen was subject to 10 consecutive sonic tests with only a short pause in between each. The peak power was recorded for each test and compared between the various materials. The purpose was to test for any "settling effects" that might be present, and determine which material shows the best testing repeatability without removal of the test specimen.

The second test was to examine the materials' response under intense sonic IR inspection parameters. For this test, each material was subject to an insonation period of $500 \mathrm{~ms}$ and a pneumatic pressure of $15 \mathrm{psi}$ while in direct contact with an unmodified flat horn tip. No aluminum test sample was in-between the horn tip and the backing material, thus the reason for the use of the flat unmodified tip. This was done to ensure even loading on the material and that only the region of interest would be in contact with the horn tip. Visual observation of the effects on the material were recorded.

\section{RESULTS}

\subsection{Material testing results}

Seven different backing materials were tested to determine the "settling effect" related to each material. We define settling effect to be the increase in peak power delivered by the ultrasonic source for sequential insonation without removing and repositioning the sample. This is believed to be caused by the test sample settling in the backing material, presumably due to an increase in the stiffness of the material. The various candidate materials were subjected to 10 consecutive sonic pulses, each $500 \mathrm{~ms}$ long, with a pneumatic pressure of 15 psi. Fig. 7 shows a graph of the peak power vs. sequential insonation number.

All the materials tested with the exceptions of the cork and HDPE show a settling effect that leads to repeatability difficulties. The cork tends to remain around an average value dipping both below and above that average. The HDPE also tends to remain somewhat constant without a noticeably consistent increase in peak power. It should be noted that the choice of the backing material has a profound effect on the peak power delivered by the supply, as shown by Fig. 7 . 


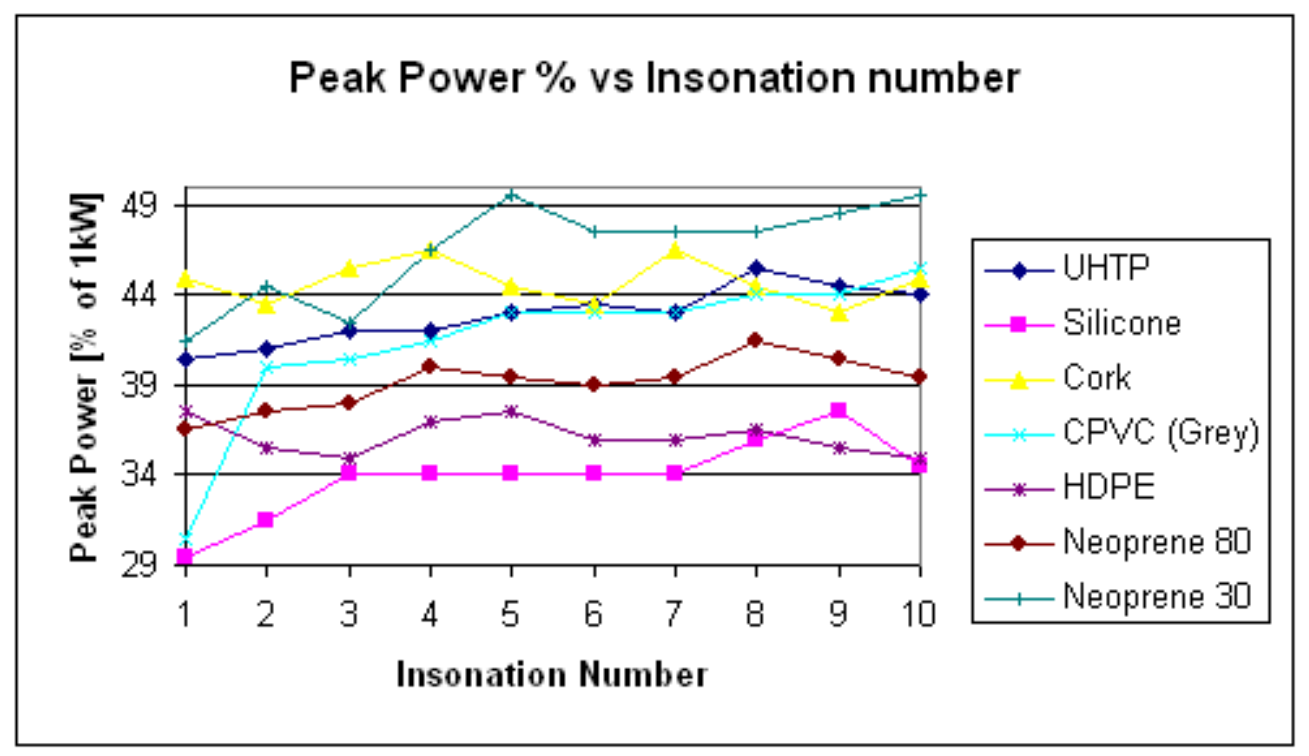

Figure 7. Plot of settling effect of various materials.

The results of the sonic IR test subjecting the materials to a sonic pulse $500 \mathrm{~ms}$ long with a pneumatic pressure of 15 psi shows the effects of the $20 \mathrm{kHz}$ vibration on the different materials as shown in Fig. 8 . The first material UHTP, formed a sticky liquid residue around the region of where it was insonated. In addition a short, through-thickness split in the material formed in the center of the horn tip contact area. The silicone also formed a sticky residue and had three splits in the material at the horn tip contact location. However the split did not go completely through the material. The cork did not split or form any type of residue, however a noticeable indentation (compaction) occurred where the horn tip made contact with the cork. The CPVC both melted and burned at the location of insonation. The HDPE had some marring at the contact point and some very light melting at the edge of the horn tip location but, in comparison to the other material tested, very little damage was observed. The 80-durometer neoprene formed significant pits and also created two types of residues, both a sticky liquid and a powder. During this test smoke from contact point was observed. The 30-durometer neoprene also formed pits and liquid residue but did not form a powder residue.
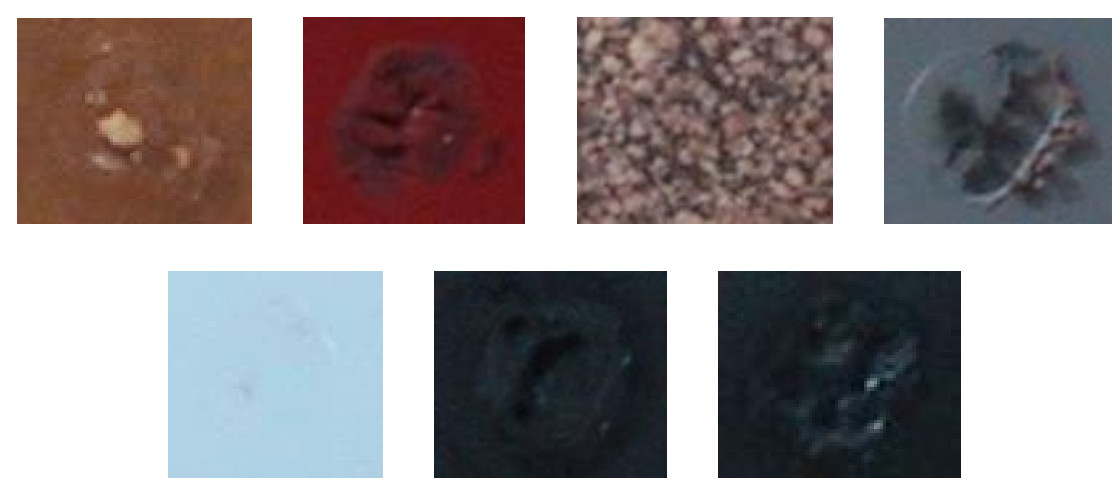

Figure 8. Left to right: UHTP, Silicone, Cork, CPVC, HDPE, Neoprene 80 Durometer, Neoprene 30 Durometer. 


\subsection{Sonic crack growth results}

Fatigue cracks were grown in the ESET sample specimen using a MTS machine with constant load amplitude of 598.9lbf with a load ratio, $\mathrm{R}=0.1$. The crack growth rates of this sample are not unusual and correspond well with published data for 2024-T3 aluminum as seen in Fig. 9.

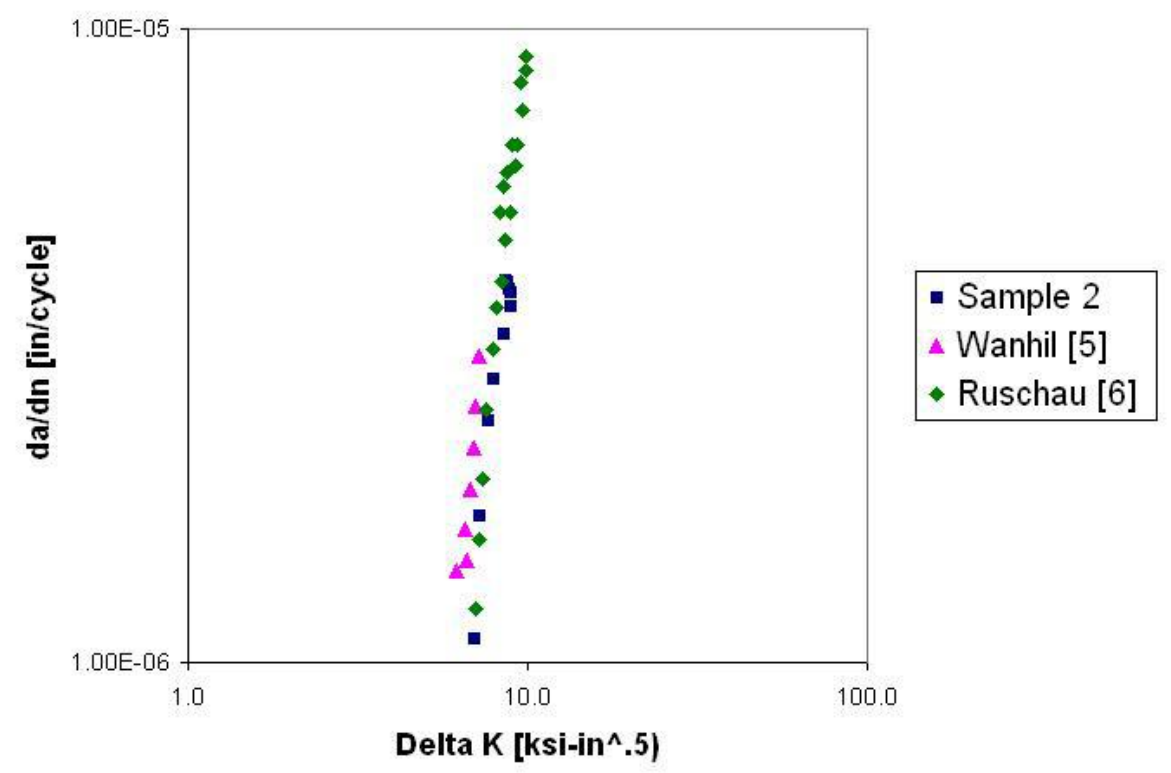

Figure 9. Crack growth rates for the 2024-T3 aluminum with $\mathrm{R}=0.1$.

Fatigue cracks were grown on three samples and each was subjected to sonic IR inspection with backing material only directly behind the area that the horn tip contacts. The pneumatic pressure was set to three different pressures resulting in nominal peak power outputs of $590 \mathrm{~W}, 675 \mathrm{~W}$, and $940 \mathrm{~W}$. Each sample was subject to a $250 \mathrm{~ms}$ sonic pulse, then removed from the template holder, and optically measured on each side to obtain an average crack length. The backing material used was UHTP due to its resistance to becoming compressed. Settling was not an issue for these tests since each sample was removed after each sonic pulse. For these tests the crack grew with each sonic pulse as seen in Fig. 10. This figure shows the total (cumulative) amount of energy delivered to the handgun after each test and the crack length at that point. It should be emphasized that the total energy calculated is that delivered by the supply to the handgun, while the actual energy delivered to the sample cannot be determined in our set-up. 


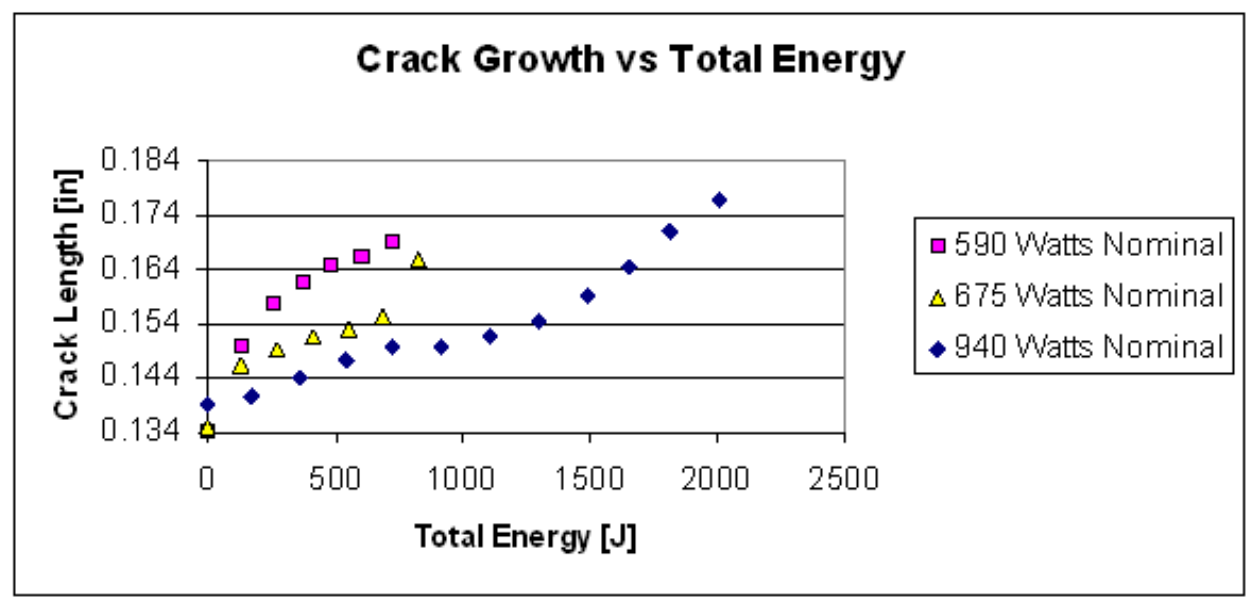

Figure 10. Crack growth vs. total sonic energy delivered to the handgun.

A micrograph was taken of the sample that corresponds to the $940 \mathrm{~W}$ test after its $10^{\text {th }}$ insonation and compared to its pre-sonic IR micrograph (Fig. 11). It can visually be seen that significant crack growth has occurred.

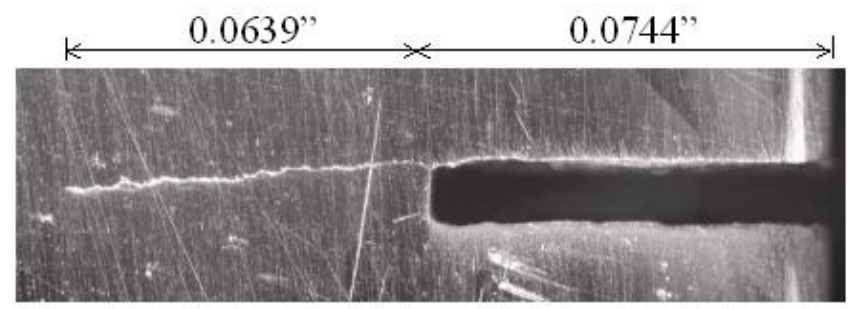

Initial Crack

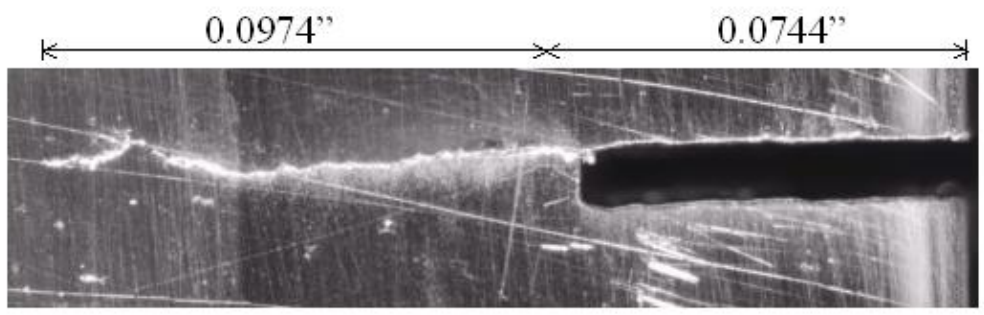

Crack after multiple insenations

Figure 11. Before and after micrograph of crack length after multiple insonations.

To determine if the crack caused by the sonic energy affects cyclic loading crack growth, a sample was loaded into the MTS machine and a crack was grown in the sample. After the crack grew to a predetermined length, it was removed from the MTS machine and subjected to multiple sonic IR tests. The sample was then placed back in the MTS machine for further cyclic loading and crack growth. The same loading parameters were used as before. Charting the progress shows that after the sonic IR-induced crack growth (the initial crack of 0.137 in. length grew to 0.183 in. after the sonic IR test), crack retardation occurred. Addition cyclic crack growth was not observed until 3000 cycles later, as shown in Fig. 12. 


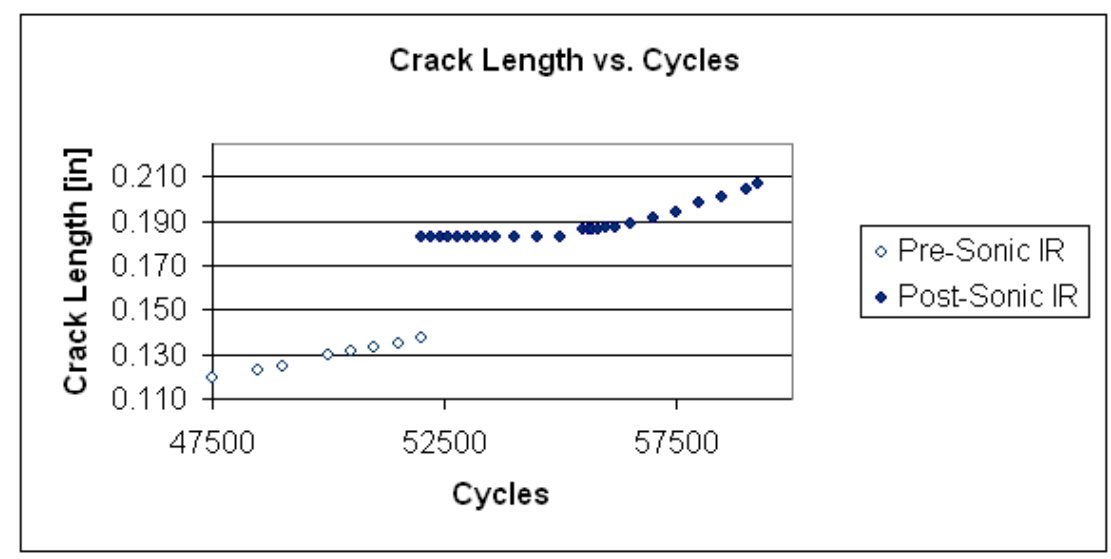

Figure 12. Crack length vs. cycles before and after sonic crack growth.

\section{CONCLUSIONS}

The choice of backing material is important when conducting sonic IR tests, as is careful control and monitoring of the holding pressure applied to the ultrasonic source. A variety of materials were tested for a settling effect and the effect of high power sonic energy on the material. The settling effect is apparent in all the materials tested with exception to cork and HPDE. This leads us to conclude that either of these two material is the best choice among the ones tested. The tendency of the cork to compress and stay compressed after repeated sonic insonation, however, suggests that it may not be a suitable backing material unless it is replaced frequently. The cork compression observed can lead to long samples (such as that used in our tests) lifting off of the backing material at the opposite end from where the handgun is located. This liftoff allows the sample to vibrate freely without any additional damping due to the backing material and can cause unpredictable and dramatic crack growth. The HPDE did not exhibit any significant settling effect nor did it grossly deform during the long duration sonic tests. It did however melt a little and did appear to have scratches in the region of the horn tip. All of the other backing materials tested exhibited settling behavior. In addition, under intense sonic energy, these materials melt, crack, or give off some residue. Based on these observations, it seems that HDPE is the best choice for backing material to give consistent and repeatable results.

Sonic IR can cause cracks to grow under typical testing conditions. It should be noted that our observation of crack growth is particular to the sample size, geometry and material that we chose. It would be extremely difficult to extrapolate our findings to other conditions at this point. In the event that the crack does propagate due to the sonic IR inspection, however, we observed that retardation occurs in subsequent crack growth through cyclic loading. The theory behind fatigue crack growth is well known under cyclic tension loading conditions, that is mode 1 loading. If a fatigue crack was being grown under cyclic tension conditions, and then is subjected to a cycle of stress much greater than those previously growing the crack, an overload effect will occur and crack growth under the previous conditions would be retarded and not grow at the same rate. However the mechanism for crack growth under sonic IR conditions is not known. It is possible that the crack growth is actually the result of a combined mode 1,2, and 3 loading but its not known if an overload occurred. It has been shown that if a crack is extended when subjected to sonic IR, subsequent mode 1 loading will exhibit crack growth retardation. At this point it is not known if crack retardation will still occur if no crack extension occurs due to the sonic IR testing. This will be determined in future research. 


\section{REFERENCES}

1. L.D Favro, Xiaoyan Han, Zhong Ouyang, Gang Sun, Hua Sui, and R.L. Thomas "Infrared imaging of defects heated by a sonic pulse", Review of Scientific Instruments, Vol. 71, p2418-2421, June 2000.

2. Thomas et al. Infrared Imaging of Ultrasonically Excited Subsurface Defects in Materials. U.S. Patent 6,236,049. May 22, 2001.

3. Zweschper, T., Riegert, G., Dillenz, A. and G. Busse. "Ultrasound Burst Phase Thermography (UBP) for Applications in the Automotive Industry", Review of Progress in Quantitative Nondestructive Evaluation. Vol. 22. AIP Conference Proceedings, Volume 657, pp. 531-536 (2003).

4. ASTM E647-00, "Standard Test Method for Measurement of Fatigue Crack Growth Rates" ASTM International.

5. Wanhill, R. J. H., "Low Stress Intensity Fatigue Crack Growth in 2024-T3 and T351", Engineering Fracture Mechanics, Vol. 301988.

6. Ruschau, J. J., "The Effect of Negative R-Ratio on Fatigue Crack Growth Rate Properties for Aluminum 7075-T73 and 2024-T3", University of Dayton Research Institute, Dayton, OH, Contract No. F33615-78-C-5002, Technical Memorandum UDR-TM-80-07, (March 1980). 